\title{
ACOUSTIC CONDUCTANCE OF A THICK-WALLED ANISOTROPIC SPHERICAL SHELL SUBMERGED IN LIQUID
}

\author{
Valery Polyakov ${ }^{1}$, Rafał Chatys ${ }^{2}$ \\ ${ }^{1}$ Institute of Polymer Mechanics, Latvian University, 23 Aizkraukles St., 1006 Riga, Latvia \\ ${ }^{2}$ Faculty of Mechatronics and Machine Building, Kielce University of Technology, Al. Tysiaclecia P.P.7, \\ 25-314 Kielce, Poland \\ E-mail: chatys@tu.kielce.pl (correspondingauthor)
}

Received 01 October 2013; accepted 28 February 2014

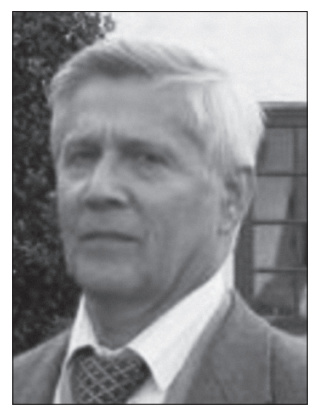

Valery POLYAKOV, PhD, Eng.

Date of birth: 1941.

Education: MSc degree in the Faculty of Mathematics and Mechanics of the State University in Leningrad in 1963, 1992 (Cand. Eng. Sci 1971) - PhD at the Latvian Academy of Sciences.

Affiliations and functions: Res. Associate, leading researcher (1986) at the Institute of Latvian University, the Institute of Polymer Mechanics.

Research interests: concerned with the problems of modeling of spatially reinforced composites (3D and 4D) composites, ultimate state of structural elements, distinctly heterogeneous beams and spherical shells subjected to concentrated loading, wave vibrations and edge effects.

Publications: author and co-author of over 80 works published in national and foreign professional journals, co-author of three monographs.

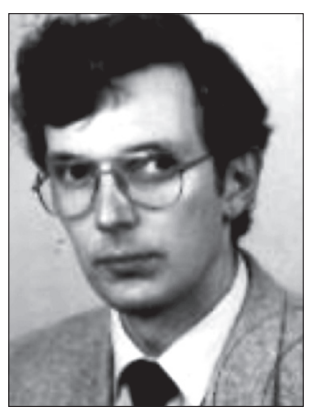

Rafal CHATYS, PhD, Eng.

Date of birth: 1970.

Education: MSc degree in Mechanical Engineering, Riga Institute of Civil Aviation Engineers in 1994, 1998 - PhD at Riga Aviation University, the Chair of the Structure and Strength of Aerial Vehicles.

Affiliations and functions: Assistant Professor at the Department of Computing and Armament (the Faculty of Mechatronics and Machine Building) at Kielce University of Technology.

Research interest: problems of mechanics of composite materials and methods for forecasting fatigue properties of polymer composites.

Publications: author and co-author of over 92 works published in national and foreign professional journals.

\begin{abstract}
In order to provide knowledge on the intensity of sound transmission through the whole thickness of an isotropic spherical shell its vibrations are resolved on the radius. The vibrations are caused by harmonic sources located on the inner surface. The relative vibration speed of the outer surface, which emits radial waves into the environment, was found. The analytical solution considers the contact interaction between the outer surface of the shell and the surrounding liquid. The method, which directs the Laplace transformations, enables to obtain a general solution of the wave problem for vibrations on the surface. The solution of the natural vibration equation and the expression of relative amplitude at non-resonance and resonance vibrations of the sphere have been investigated with regard to radial and circumferential elastic modulus as well as the relative parameters of surface thickness.
\end{abstract}

Keywords: sphere vibrations, isotropic material, frequency, Laplace method, complex solution, amplitude. 


\section{Introduction}

The feature of employing dynamic characteristics is of importance in developing test models for anisotropic materials (Rytov 1956; Lasn et al. 2011), along with the averaging and homogenization methods (Barski, Muk 2011) for structurally inhomogeneous materials. In this relation, it must be noted that the dynamic solutions in the case of a complicated deformation mechanism of materials that takes into account the interaction between elastic, piezoelectric, and dielectric properties require further modifications of material models (Lagzdin et al. 2013). They include calculation models for revealing the relation between the eigenfrequency spectrum of anisotropic plates and the piezoelectric effect in the case of different boundary-value problems (Narita 2003). The structural models of a medium, which take into account the effect of lamination on the dispersion of wave vibrations or the porosity on their damping, are rather intricate for the calculation of its dynamic behavior because of the multi-parametric relations in the search for a solution. Therefore, to deduce the solutions to wave problems and to select an optimum one from them, in particular, for the problems of acoustic vibrations in solid layers submerged in liquid, calculation approximations including nonstandard approaches, such as the genetic algorithm, are employed (Daneshjou et al. 2011).

The study assesses the capability of the operational method to implement boundary problem solving and to develop calculation models for dynamic characteristics of construction elements in which new composite materials are used.

A more precise analytical analysis of the relationship among the amplitude, the physical parameters and the intrinsic geometry of the solid body requires the use of appropriate solutions. However, calculations in the case of a combination of homogenous and inhomogeneous problems at dynamic loading can be difficult due to the anisotropy of elastic properties and the form of the region where the solution is given. The use of the Fourier (Brekhovskikh, Goncharov 1982) or Laplace (Lavrentyev, Shabat 1973) transformation methods (applying a function of a complex variable) provides the possibility to present the solutions to boundary-value problems in simple series in terms of eigenvalues and to extend them to the cases of non-stationary wave processes. In the present study, a relative change in the amplitude as a function of model parameters has been obtained using the Laplace method. A non-stationary solution, taking into account the theory of functions of a complex variable (Lavrentyev, Shabat 1973), is considered for the case of resonant coincidence of the disturbance and the first frequencies of waves.

\section{Basic relations for the radial vibrations of the spherical model}

The acoustic effect on the surrounding liquid (or gaseous) medium of a spherical shell's vibrating surface is described by the intensity of radiation associated with the normal speed of its outer surface. The relation of speed caused by the action of a time-sinusoidal source of motion of the sphere's inner surface, for the boundary conditions accepted, was found according to the method described in (Polyakov 2012a, b). A certain relation also exists among the physical material parameters of the sphere, the relative thickness of its wall and the individual frequencies determined according to the boundary conditions. The relative speed of the outer surface of the sphere in the considered boundary problem is characterized by the fact that it is obtained without limits to the thickness of the sphere as a shell and, at the same time, without the simplifications of the Kirchhoff Love deformation kinematics of vibrations of plates and shells. The limitation of the general model is related to its one-dimensional approach with regard to the space in the tests of vibrations along the radius. However, such a simplification enabled to produce approximate formulas of the parametric analysis for the selected analytical approach. The final expression has been derived for the relative speed values of the points on the sphere's outer surface, in this way:

$$
\begin{aligned}
& \frac{v_{\text {out }}}{v_{\text {inn }}}=\tilde{v}(t)=\operatorname{Re} \frac{\sqrt{\varepsilon e^{i \varpi\left(\frac{c}{R_{0}}\right) t}}}{N_{\mu}(i \varpi)}+ \\
& 2 \operatorname{Re} \sqrt{\varepsilon} \sum_{k=1}^{\infty} \frac{\lambda_{k} e^{\lambda_{k}\left(c / R_{0}\right) t}}{\left(\lambda_{k}^{2}+\varpi^{2}\right) N_{\mu}^{\prime}\left(\lambda_{k}\right)}
\end{aligned},
$$

in which, the imaginary eigenvalues $\lambda_{k}, k=1,2, \ldots$, and the dimensionless material parameter $\varpi$ are connected with the frequencies of natural $\omega_{k}$ and forced $\omega$ vibrations by the external radius $R_{0}$ of the sphere, and sound speed $\mathrm{c}$ according to the following relations:

$$
\begin{aligned}
& \left|\lambda_{k}\right|=\frac{\omega_{k} R_{0}}{c}, \varpi=\frac{\omega R_{0}}{c}, \alpha=\frac{c}{c_{0}}, \\
& \gamma=\frac{\rho}{\rho_{0}}, \varepsilon=\frac{R_{1}}{R_{0}} .
\end{aligned}
$$

The remaining parameters in Eq. (2) express the ratios of sound speeds in the sphere and liquid, their specific densities, and the radii of the hollow and the outer surface, respectively. At given values of these parameters, the first of the parameters, which determines the eigenfrequencies, in (2) is found from the equation below:

$$
\begin{aligned}
& \left(\chi-\frac{x^{2}}{\gamma}\right)\left[J_{\mu}(\varepsilon x) Y_{\mu}(x)-J_{\mu}(x) Y_{\mu}(\varepsilon x)\right]+ \\
& x\left[Y_{\mu-1}(x) J_{\mu}(\varepsilon x)-Y_{\mu}(\varepsilon x) J_{\mu-1}(x)\right]=0 .
\end{aligned}
$$


Both real and simple roots of Eq. (3), $x=\lambda_{\mathrm{k}}, \mathrm{k}=1$, $2, .$. , also depend on the parameter $\chi$ of the boundaryvalue problem and the order $\mu$ of Bessel functions, which give the general solution for the problem considered. The relations between the previously mentioned parameters and the elastic constants are:

$\mu=\sqrt{\frac{1}{4}+\frac{2\left(1-v_{\perp r}\right)}{1+v_{\perp \perp}} \frac{E_{\theta}}{E_{r}}}, \quad \kappa^{2}=\sqrt{\frac{1-v_{\perp r}}{1+v_{\perp \perp}} \cdot \frac{E_{\theta}}{E_{r}}}, \quad \chi=-\mu-\frac{1}{2}+\frac{2 \kappa^{2} v_{\perp r}}{1-v_{\perp r}},(4)$

where the Poisson ratio $v_{\perp r}$ characterises the transverse contraction of the material under tension along the radius.

Expression (1) for the vibration speed of the sphere's outer surface contains various vibration amplitudes. It should also be noted here that, in essence, all coefficients of the trigonometric functions are residues in a complex representation of speed function (1). It stems from the theorem of summation of residues in the entire complex plane, including the residue at the infinite point, where $\mathrm{k} \rightarrow \infty$, and their sum is zero. According to Lavrentyev, Shabat (1973) we have:

$$
\begin{aligned}
& \operatorname{res} f\left(\lambda_{0}\right)+\operatorname{res} f\left(\lambda_{1}\right)+\ldots+\operatorname{res} f\left(\lambda_{n}\right)+ \\
& \operatorname{res} f(\infty)=0
\end{aligned}
$$

The initial residue is calculated at the pole $\lambda_{0}=i \varpi$. Assuming that the last residue of the sum (1) at the pole $\lambda_{k}=i \infty$ is equal to zero, it can be found according to Eq. (5) that:

$$
\tilde{v}(t)=2 \sqrt{\varepsilon} \operatorname{Re} \sum_{k=1}^{n} \frac{\lambda_{k}\left[\cos \left(t\left|\lambda_{k}\right| c / R_{0}\right)-\cos \left(\varpi t c / R_{0}\right)\right]}{\left(\lambda_{k}^{2}+\varpi^{2}\right) N_{\mu}^{\prime}\left(\lambda_{k}\right)} .
$$

In the case of resonance for any mode when $\varpi \rightarrow\left|\lambda_{k^{\prime}}\right|$, according to L'Hospital's rule, the following time dependence is obtained:

$$
\begin{aligned}
& \tilde{v}(t)=\frac{t c \sqrt{\varepsilon}}{R_{0}\left|\lambda_{k^{\prime}}\right|} \operatorname{Re}\left[\frac{\lambda_{k^{\prime}}}{N_{\mu}^{\prime}\left(\lambda_{k^{\prime}}\right)}\right] \sin \left(\frac{t\left|\lambda_{k^{\prime}}\right| c}{R_{0}}\right)+ \\
& 2 \sqrt{\varepsilon} \operatorname{Re} \sum_{k=1}^{n} \frac{\lambda_{k}\left[\cos \left(t\left|\lambda_{k}\right| c / R_{0}\right)-\cos \left(t\left|\lambda_{k^{\prime}}\right| c / R_{0}\right)\right]}{\left(\lambda_{k}^{2}+\left|\lambda_{k^{\prime}}\right|^{2}\right) N_{\mu}^{\prime}\left(\lambda_{k}\right)},
\end{aligned}
$$

where the prime at the summation sign means that $k \neq k^{\prime}$.

A further analysis of the vibration speed of the sphere's external surface is carried out relative to the parameters of relationships (1), (6), and (7). The relative speed according to the quasi-static model, when the inertial additive is neglected in the equation of motion, depends on two model parameters $\mu$ and $\varepsilon$ :

$$
\tilde{v}\left(R_{0}, t\right)=\frac{2 \sqrt{\varepsilon} \cos (\omega t)}{\varepsilon^{-\mu}+\varepsilon^{\mu}}
$$

and coincides with the calculations obtained from (1) as shown in (Polyakov 2012a) at a great value of the parameter $E_{\theta} / E_{r}>>1$.

\subsection{Examples of amplitude calculations and the approximation of time-dependent relations}

The calculated values of the coefficients of exponents in series (1), including the free term, are equal to the relative amplitudes of the vibration modes at $r=R_{0}$. Their sum is equal to the relative value of the vibration speed on the sphere's external surface at the point in time where $t=0$. At a finite number of terms of sum (1), condition (5) can serve as a criterion for accuracy of the choice of this number, i.e. it can show the variation of a particular sum from a zero. Figure 1 illustrates the variation in the values of the first four terms at the onset of the wave process as a function of parameter $\beta$, which shows the degree of anisotropy of the sphere's elastic properties. The initial value, $\beta=1$, corresponds to an isotropic material. The sharp drop in the values of all coefficients at $\beta>50$ agrees with the growth in eigenfrequencies, as follows from (Polyakov 2012b). It should be noted that, at a low degree of anisotropy, neither a forced vibration alone, nor the simultaneous account of the first natural mode allows us to regard the calculated approximation of the vibration amplitude as sufficiently accurate when considering the sphere's thickness, when $\mathrm{R}_{1} / \mathrm{R}_{0}=0.50$. The computation of three natural vibration modes led to a variation of sum (1) from zero within the limits of $2 \%$ with a light liquid surrounding the sphere, when $\gamma=400$ (Fig. 1). In the case of a heavy liquid, when $\gamma=1$, this difference increased to $5 \%$. The sum of ordinates of all four curves, at an arbitrary value of parameter $\beta$, shows the deviation of the speed from zero in an undisturbed state, as assumed in the operational transformation.

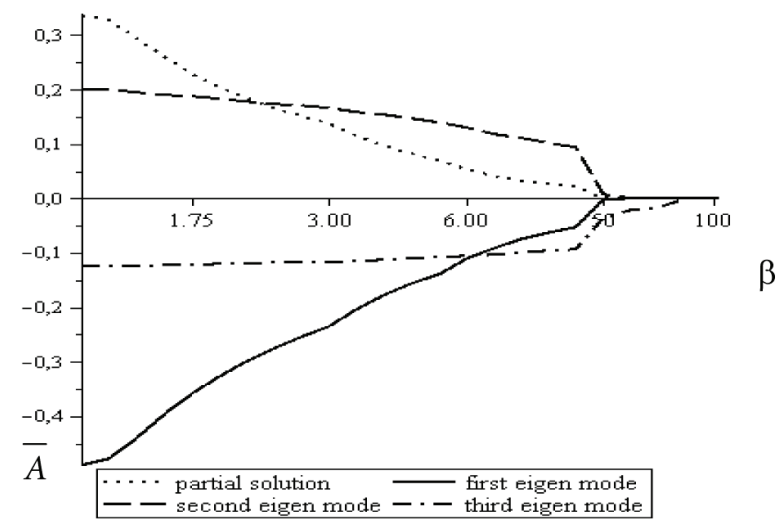

Fig. 1. Amplitude of the sphere's relative speed of external surface $r=R_{0}$ versus the anisotropy parameter $\beta=E_{\theta} / E_{r}$ for the three modes of natural vibrations and forced vibration with the relative frequency $\varpi=\lambda_{1} / 4$ at $\varepsilon=R_{1} / R_{0}=0.50, \gamma=400$

The influence of the sphere's wall thickness on the resonant speed is related to the current time by means of the linear and oscillating character of the time functions. At a fixed time variable, the dependence $\bar{v}(\varepsilon)$, in the entire spectrum of eigenfrequencies, can be regarded as, $\bar{v}\left(\lambda_{k}\right), k=1,2, \ldots$ corresponding to the assigned value 
of $\varepsilon$. The approximation of sum (7) at different values of the parameter and fixed time is presented in figure 2 .

The change in the amplitude $\bar{A}(\varepsilon)$ of the first resonant mode at the time point $t_{0}=R_{0} / c$ allows us to trace its monotonic growth, which is not connected with the time, but depends only on the growth of the function $\sqrt{\varepsilon} / N_{\mu}^{\prime}\left(\lambda_{1}(\varepsilon)\right)$. The first values of $\lambda_{1}(\varepsilon)$ corresponding to the isotropic and anisotropic spheres immersed in a light liquid are taken from Polyakov (2012b). The quasistatic model yields a relation between the vibration amplitude and the parameter $\varepsilon$ for these spheres according to Eq. (8).

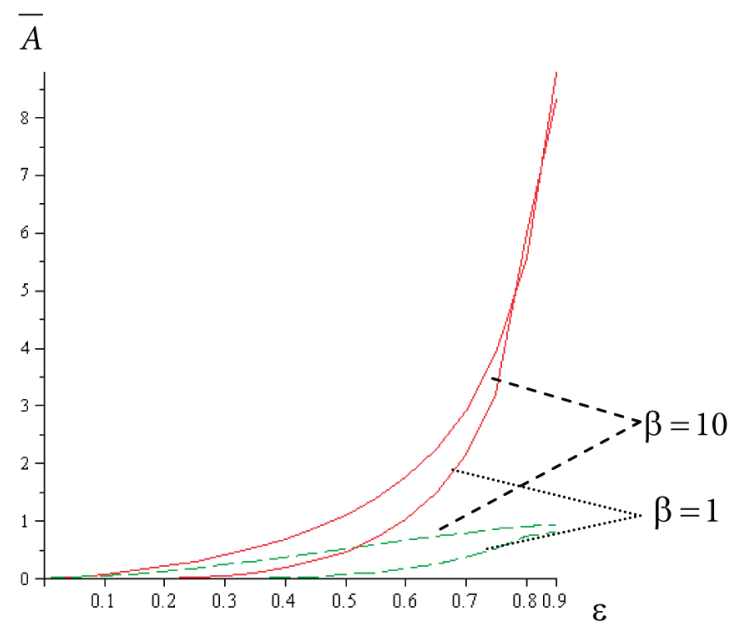

Fig. 2. The relative amplitude of the resonant mode versus the parameter $\varepsilon=\mathrm{R}_{1} / \mathrm{R}_{0}$, at $\mathrm{t}_{0}=\mathrm{R}_{0} / \mathrm{c}$ and $\varpi=\lambda_{1}$ for isotropic $(\beta=1$ and $v=0.3)$ and anisotropic $(\beta=10)$ spheres in a light liquid $(\gamma=400)$. Dashed lines indicate variations in the stationary amplitude according to the quasi-static model

\section{Conclusions}

Based on the analysis performed on the sphere the amplitudes of the forced and resonant vibrations were obtained analytically. As a result, the amplitude of wave radiation from the surface of the sphere into the surrounding liquid was evaluated. It was found that lowering the wall thickness through a growth in the parameter $\varepsilon=\mathrm{R}_{1} / \mathrm{R}_{0}$, increases the oscillation amplitude of speed. The most significant change of amplitudes by virtue of the thick-wall sphere theory is observed in the interval $\varepsilon \in(0.20-0.75)$. As a restriction on the highest amplitude in a non-resonant mode, it is possible to use its dependence on the parameter $\varepsilon$ obtained for the quasistatic model. With an increase in the degree of anisotropy of the sphere's material (through an increase in the $\beta$ parameter) the vibration amplitude decreases more than with an order of magnitude (at $\beta>50$ ) in comparison with isotropic materials. The increase in the amplitude on $t$ can be described with one element of the complete equation (7).

\section{References}

Daneshjou, K.; Ramezani, H.; Talebitooti, R. 2011. Wave transmission through laminated composite double-walled cylindrical shell lined with porous materials, Applied Mathematics and Mechanics 32(6): 1-16.

Narita, Y. 2003. Layerwise optimization for the maximum fundamental frequency of laminated composite plates, Journal of Sound and Vibration 263: 1005-1016.

Barski, M.; Muk, A. 2011. Metody gomogenizatsii dlya dvukhfaznykh kompozitov [Homogenization methods for twophase composites], Mekhanika Kompozitnykh Materialov [Mechanics of Composite Materials] 47(4): 557-566 (in Russian).

Brekhovskikh, L. M.; Goncharov, V. V. 1982. Vvedeniye v mekhaniku sploshnykh sred ( $v$ prilozhenii $k$ teorii voln) [Introduction to continuum mechanics (in the annex to the theory of waves)]. M.: Nauka. 334 s. (in Russian).

Lavrentyev, M. A.; Shabat, B. V. 1973. Metody teorii funktsiy kompleksnogo peremennogo [Methods of complex variable theory]. М.: Наука. 737 с. (in Russian).

Lagzdin, A.; Zilauts, A.; Beverte, I., et al. 2013. Raschet konstant uprugosti vysokoporistogo poroplasta s oriyentirovannoy strukturoy [Calculation of the elastic constants of highly porous cellular plastic-oriented], Mekhanika Kompozitnykh Materialov [Mechanics of Composite Materials] 49(2): 181-192 (in Russian).

Lasn, K.; Klauson, A.; Shati, F., et al. 2011. Eksperimentalnoye opredeleniye uprugikh konstant ortotropnoy kompozitnoy plastiny pri pomoshchi voln Lemba [Experimental determination of elastic constants of orthotropic composite plates using Lamb waves], Mekhanika Kompozitnykh Materialov [Mechanics of Composite Materials] 47(4): 617-632 (in Russian).

Polyakov, V. A. 2012a. Akusticheskaya provodimost poloy anizotropnoy sfery, pogruzhennoy $\mathrm{v}$ zhidkost. 1. Volnovaya model kontaktnogo vzaimodeystviya tverdoy poloy sfery $\mathrm{i}$ zhidkosti. Vyrozhdennyye resheniya [Acoustic conductivity of anisotropic hollow sphere immersed in liquid. 1. Wave model of contact interaction of solid and hollow sphere liquid degenerate solutions], Mekhanika Kompozitnykh Materialov [Mechanics of Composite Materials] 48(4): 653-678 (in Russian).

Polyakov, V. A. 2012b. Akusticheskaya provodimost poloy anizotropnoy sfery, pogru $\neg$ zhennoy v zhidkost. 1. Volnovaya model kontaktnogo vzaimodeystviya tverdoy poloy sfery $\mathrm{i}$ zhidkosti. Vyrozhdennyye resheniya [Acoustic conductivity of anisotropic hollow sphere immersed in liquid. 1. Wave model of contact interaction of solid and hollow sphere liquid degenerate solutions], Mekhanika Kompozitnykh Materialov [Mechanics of Composite Materials] 48(5): 807-824 (in Russian).

Rytov, S. M. 1956. Akusticheskiye svoystva melkosloistoy sredy [Acoustic properties of fine-layered medium], Akustich. Zhurnal 2(1) (in Russian). 